SENSITIVITY OF PBX-9502

AFTER RATCHET GROWTH

R. N. Mulford, D. C. Swift

November 21, 2011

American Physical Society Topical Conference on Shock Compression of Condensed Matter Chicago, IL, United States

June 26, 2011 through July 1, 2011 
This document was prepared as an account of work sponsored by an agency of the United States government. Neither the United States government nor Lawrence Livermore National Security, LLC, nor any of their employees makes any warranty, expressed or implied, or assumes any legal liability or responsibility for the accuracy, completeness, or usefulness of any information, apparatus, product, or process disclosed, or represents that its use would not infringe privately owned rights. Reference herein to any specific commercial product, process, or service by trade name, trademark, manufacturer, or otherwise does not necessarily constitute or imply its endorsement, recommendation, or favoring by the United States government or Lawrence Livermore National Security, LLC. The views and opinions of authors expressed herein do not necessarily state or reflect those of the United States government or Lawrence Livermore National Security, LLC, and shall not be used for advertising or product endorsement purposes. 


\title{
SENSITIVITY OF PBX-9502 AFTER RATCHET GROWTH
}

\author{
R. N. Mulford ${ }^{1}$ and D. C. Swift ${ }^{2}$ \\ ${ }^{1}$ Los Alamos National Laboratory, Los Alamos NM 87545 \\ ${ }^{2}$ Lawrence Livermore National Laboratory, Livermore, CA 94550
}

\begin{abstract}
Ratchet growth, or irreversible thermal expansion of the TATB-based plastic-bonded explosive PBX-9502, leads to increased sensitivity, as a result of increased porosity. The observed increase of between 3.1 and 3.5 volume percent should increase sensitivity according to the published Pop-plots for PBX-9502 [1]. Because of the variable size, shape, and location of the increased porosity, the observed sensitivity of the ratchet-grown sample is less than the sensitivity of a sample pressed to the same density. Modeling of the composite, using a quasi-harmonic EOS for unreacted components [2] and a robust porosity model for variations in density [3], allowed comparison of the initiation observed in experiment with behavior modeled as a function of density. An Arrhenius model was used to describe reaction, and the EOS for products was generated using the CHEETAH code [4]. A 1-D Lagrangian hydrocode was used to model in-material gauge records and the measured turnover to detonation, predicting greater sensitivity to density than observed for ratchet-grown material. This observation is consistent with gauge records indicating intermittent growth of the reactive wave, possibly due to inhomogeneities in density, as observed in SEM images of the material [5].
\end{abstract}

Keywords: ratchet growth, TATB, PBX-9502, explosive initiation.

PACS: 65.40.De, 62.50.Ef, 82.40.Fp, 82.33.Vx

\section{INTRODUCTION}

Ratchet growth of TATB and TATB-based plastic bonded explosives is of interest both as a practical concern in evaluating the safety of thermally cycled explosives, and as a unique behavior among explosive compounds. Hysteretic expansion of the solid occurs on thermal cycling, with the total volume increasing on repeated cycles, asymptotically approaching a maximum. Ratchet growth is observed in graphitic materials such as TATB, where large flat crystals are present, and where anisotropic expansion on crystallographic axes requires rearrangement of crystal packing at elevated temperature. This rearrangement is preserved to some degree upon cooling, resulting in net expansion of the solid. Thermal expansion along the crystallographic axes of TATB vary widely: values of $248,8.3$ and 21 $\mathrm{nm} / \mathrm{mm} \cdot \mathrm{K}$ have been measured for the $a, b$, and $c$ axes of the monoclinic TATB crystal [6]. The $a$ axis perpendicular to the molecular plane is generally the short axis of crystallites of TATB. The coefficient of thermal expansion for the bulk is $50 \mathrm{~nm} / \mathrm{mm} \cdot \mathrm{K}$.

Irreversible thermal expansion is amplified in pressed solids, as plate-like crystallites align perpendicular to the pressing axis, creating an anisotropic solid. Crystallites may be bent or strained during pressing, creating impetus for relaxation during heating, although the bonding agent may ameliorate the expansion by pulling the crystallites back into their original positions on cooling. Heating above the glass-transition temperature allows the bonding agent to flow, permitting an expanded arrangement of crystallites to be preserved by the bonding agent on cooling, creating an additional mechanism to increase volume. Decomposition of the binder reduces the restoring forces that preserve the original volume. 
A number of studies have been made of the phenomenon and its consequences for the mechanical and reactive behavior of TATB and TATB-based explosives. Early studies by Cady [7] were supplemented by detailed experiments at temperatures relevant to environmental exposures [8], and complete characterization of pore sizes in TATB and LX-17 [9].

\section{EXPERIMENTAL PROCEDURE}

A ram-pressed billet of PBX-9502 was machined into 2"x 2" cylinders, and thermally cycled from room temperature to $140^{\circ} \mathrm{C}$ in vacuum, heating at $2^{\circ} \mathrm{C}$ and cooling at $2.5^{\circ} \mathrm{C}$. Faster rates of heating and cooling produced cracking in the cylinder. Evolved gases were collected. Dimensions were measured after each thermal cycle, at multiple locations on each cylinder.

After expansion, cylinders were remachined to be suitable for gas gun impact targets, and inmaterial gauging was glued into each target [10]. Gauge configurations included particle velocity and shock-tracker gauges [11].

Targets were shocked by impact of PMMA impactors, at $1.4 \mathrm{~mm} / \mu \mathrm{sec}$, the maximum velocity attainable using the light gas gun. This single velocity was required as a safety measure to prevent any possibility of undetonated explosive powder contaminating the target chamber.

\section{MODELING}

A reactive flow model was developed for PBX-9502, based on earlier work on nitromethane, HMX, and AN-based explosives [2], Heterogeneous explosive is treated as a mixture of pure components, each with an EOS and constitutive model. Porous materials are modeled using the appropriate volume fraction of void or gas. The stress state and temperature in each component relax exponentially from the shocked state toward the average. Localized deformation, either plastic strain or viscous flow, at heterogeneities or "hot spots" increases heating. Reactions transfer material from reactant to product components according to an Arrhenius rate in which the frequency factor and energy barrier may depend on temperature and pressure. A single rate law is specified for each component, though three rates are considered: bulk, bulk pressure with temperature determined by localized deformation, and temperature and pressure defined by adjacent material. The last two terms represent hotspots.

The reaction rate for pure TATB was calibrated against run distances for shock initiation of detonation, for material of a range of porosity.

Thermodynamically consistent EOS were used for TATB, Kel-F, and reaction products of different mixtures of the reactants [13]. A quasiharmonic EOS was generated for each solid component, by adjusting the zero Kelvin isotherm [13] to finite temperature using the vibrational modes of the material. For TATB, the quasiharmonic EOS curved sufficiently that, with porous compaction, the EOS fit both low- and highpressure data without requiring a phase transition. For Kel-F, shock speed data were reproduced up to $\sim 30 \mathrm{GPa}$.

For the reaction products of TATB, Kel-F, and mixtures of the two, EOS were calculated using the thermo-chemical program CHEETAH [4].

For the heterogeneous reaction model [2], mechanical and thermal equilibration times were estimated from the grain size to be $\sim 0.1$ and $\sim 10$ $\mu \mathrm{s}$. Arrhenius parameters were calculated to reproduce the run distance [1] for pure TATB of the highest density reported, and tested by predicting the run distance for higher porosity (Fig. 1). Simulations were performed using a Lagrangian hydrocode with $0.1 \mathrm{~mm}$ cell size.

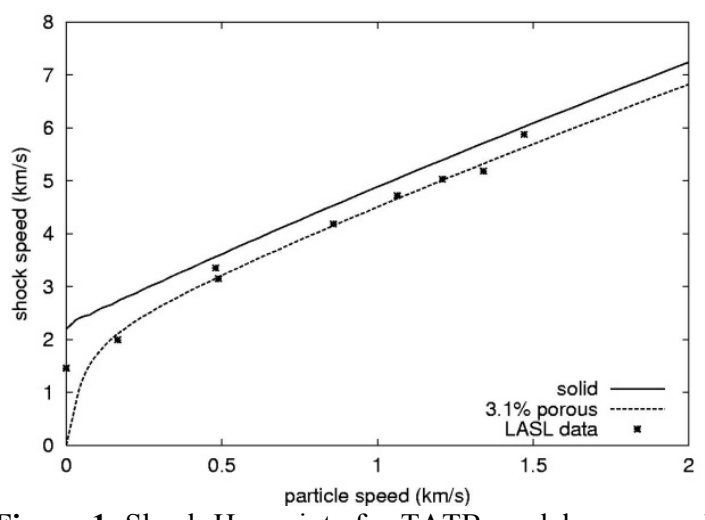

Figure 1. Shock Hugoniots for TATB model, compared with experimental data for $3.1 \%$ porous material. (Porous "snowplow" model is not valid at low pressure.)

The plastic enhancement terms and constitutive model previously developed for HMX [2] proved adequate for TATB. With plasticity, 
agreement was obtained with the experimental pressure-distance relation using low values of the parameters $\mathrm{Z}=1.0 \times 10^{4} / \mu \mathrm{s}, \mathrm{T}^{*}=9000 \mathrm{~K}$. This frequency factor is more consistent with values expected for atomic vibrations, but the barrier temperature seems unphysically low. For TATB, the calorimetry values were $\mathrm{Z}=3.18 \times 10^{13} / \mu \mathrm{s}, \mathrm{T}^{*}$ $=30157 \mathrm{~K}$ [1]. The variation of the run distancepressure relation with porosity was reproduced adequately well by the model, given the uncertainty in the experimental data (Fig. 2).

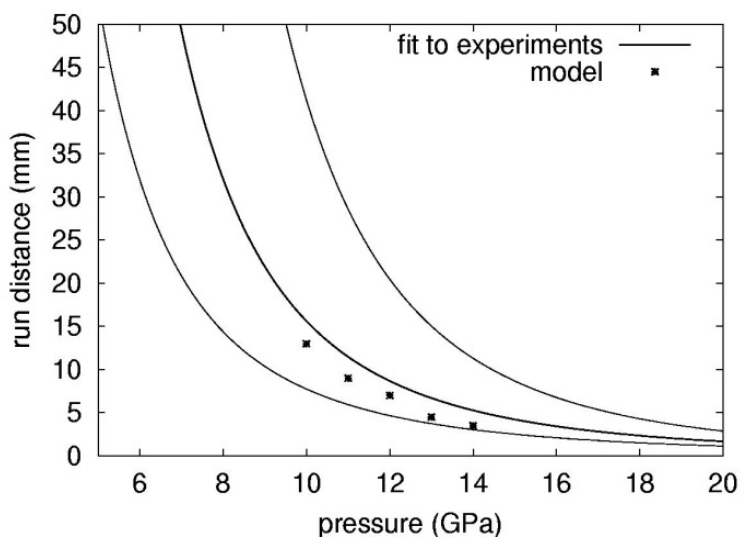

Figure 2. Single-shock run distance for PBX-9502. Fit to experimental data (lines, nominal and limits) [1], compared with calculations using temperature-dependent reactive flow model for PBX-9502 (points).

Interestingly, some of the TATB simulations exhibited a two-staged build up to detonation, as observed experimentally for micronized and ultrafine TATB [1]. For higher values of the Arrhenius parameters, the model predicted "homogeneous" shock initiation from shock heating behind the shocked surface, as opposed to "heterogeneous" build up at the shock front.

Calculations were then performed of single shock initiation in PBX-9502. A slow decomposition was included for Kel-F; the run distance was not sensitive to this rate or to the fraction of Kel-F in the gas phase. If the simplistic assumption was used that every component in the heterogeneous mixture shared surface area with every other component, the run distance predicted for PBX9502 was systematically too short. PBX-9502 is manufactured by coating the TATB grains with a
Kel-F lacquer [1], so TATB is less likely to be exposed directly to hot gas in compressed intergranular pores. The run distance was brought into better agreement by restricting the surface burn term in the TATB reaction rate to be driven by the state in the Kel-F only (Fig. 2).

\section{RESULTS AND DISCUSSION}

Ratchet grown material exhibited densities of about $1.825 \mathrm{~g} / \mathrm{cc}$, or about $94 \%$ of TMD for PBX9502. Density was determined from the exterior dimensions of the part after cooling. The part expanded asymptotically with repeated cycles, reaching near-completion after four cycles.

Initiation behavior of the ratchet-grown $\mathrm{PBX}$ 9502 was measured using in-material gauging. [10]

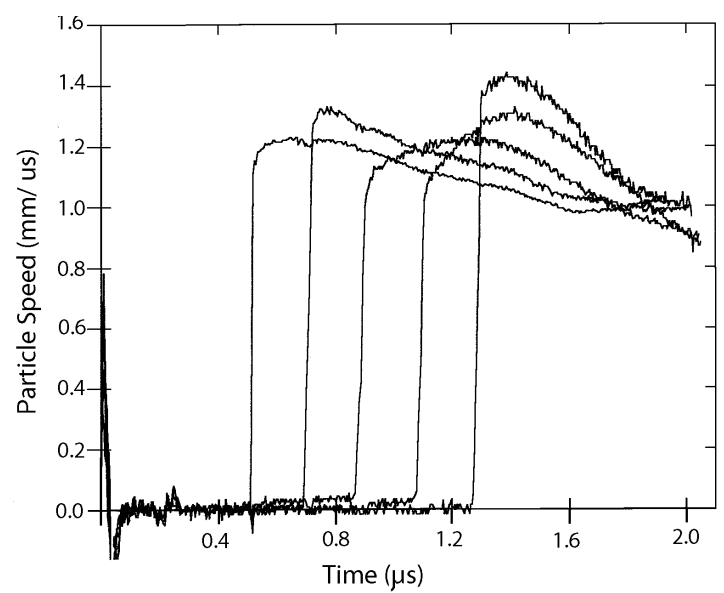

Figure 3. MIV gauge traces from shot 1010, showing development, failure, and recovery, of reactive wave.

As seen in Fig. 3, growth of the reactive wave was not uniform or simple. This behavior may be typical of the material. Gauging included a shocktracker configuration [11], which permitted relatively precise determination of transition to detonation, as shown in Fig. 4. Also seen in Fig. 4 is variation in the velocity of the reactive wave during initiation (fit to straight line.) This gauge extended further into the material than did the MIV gauges.

The run distances, pressures, and other parameters for the four shots have been described elsewhere [5]. 


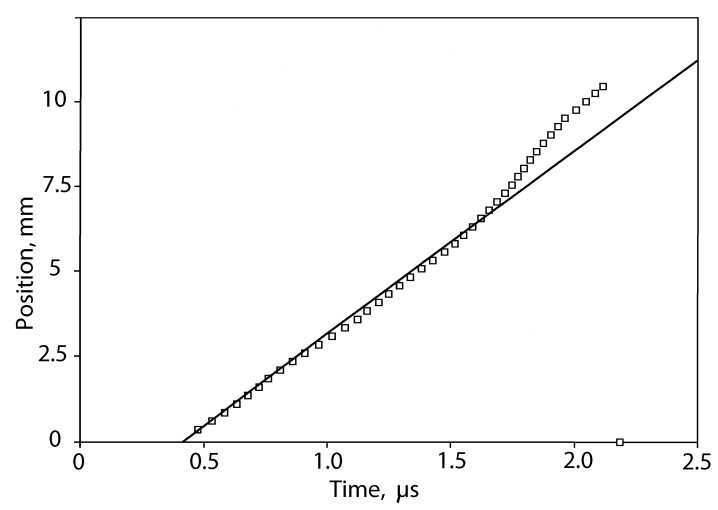

Figure 4. Shock tracker record, showing clear transition to detonation in shot 1010 .

Modeling was performed to predict sensitivity at the apparent density of the expanded samples.

Porosity was adjusted without correction to the Arrhenius factors governing the reaction. As seen in Fig. 5, the observed sensitivity of the ratchet grown material to shock initiation was somewhat lower than anticipated from modeling at the bulk density of the ratchet grown material.

Variation of the Arrhenius factor might be expected to reflect changes in inherent reactivity, such as sensitization due to partial decomposition. Changes in the Arrhenius factor had too small an effect on the sensitivity to be useful in modeling this system. This is consistent with observations of partial decomposition [14] which were observed to occur at higher temperatures (approx $250^{\circ} \mathrm{C}$.)

The prevalence of cracks in parts heated or cooled at higher rates suggests that variation of density inside the part, as seen in SEM images [5], is likely to have precluded uniform initiation.

\section{CONCLUSIONS}

This ratchet-grown material appears to undergo initiation more gradually and at a slightly higher pressure than would be anticipated for PBX9502 pressed to a similar bulk density. In-material gauging, shock-tracker records, and modeling of uniform pressings of the observed bulk density all indicate that initiation may be retarded in some cases for PBX-9502 which has undergone ratchet growth at temperatures around $140^{\circ} \mathrm{C}$. The effect is likely due to inhomogeneity in density, and is unlikely to be highly reproducible.

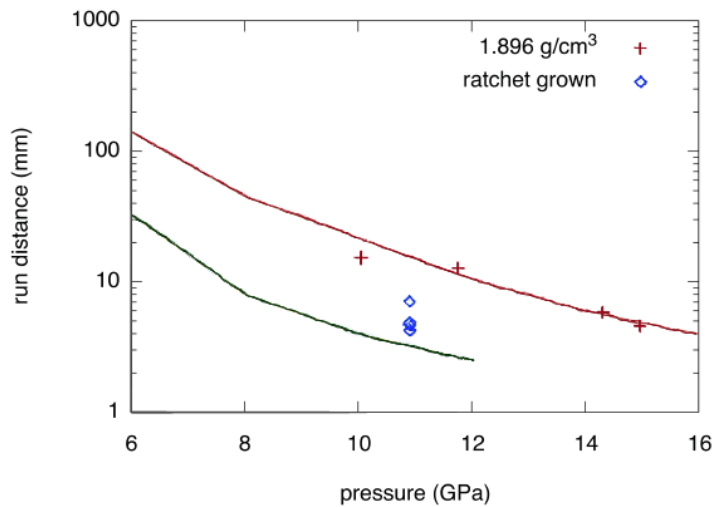

Figure 5. Pop plot for PBX-9502 before ratchet growth [1] (upper curve) compared to Pop plot adjusted to the measured bulk density of 1.825 (lower curve.) Calculated Pop plot at low density indicates longer run distance than measured for ratchet-grown material (diamonds.)

\section{REFERENCES}

1. Gibbs, T. R. and Popolato, A., "LASL Explosive Property Data" (U. Calif. Press, Berkeley, 1980).

2. Mulford, R. N. and Swift, D. C., AIP Conf. Proc. 620, pp. 415-418, (2002).

3. Mulford, R. N., Braithwaite, M., and Swift, D. C., Proc. $12^{\text {th }}$ Int. Det. Sym., held San Diego, CA, August 2002, pp. 927-936.

4. Fried, L. E., Howard, W. M., Souers, P. C., Vitello, P. A., (Lawrence Livermore National Laboratory), "CHEETAH" 3.0, software, documentation (2001).

5. Mulford, R. N., and Romero, J. A., AIP Conf. Proc. 429, pp 723-726 (1998).

6. Kolb, J. R., and Rizzo, H. F., Propell. Explos. 4, pp 10-16 (1979).

7. Howard Cady, Los Alamos National Laboratory, private communication.

8. Thompson, D. G., et al., Propell. Explos. Pyrot. 35, 507-513 (2010); Gustavsen, R. L., et al., in Proc $14^{\text {th }}$ Int. Det. Sym., Coeur d'Alene, ID, April 2010.

9. Willey, T. M., Propell. Explos. Pyrot. 31, pp 466471 (2006).

10. John Vorthman, Los Alamos National Laboratory, private communication.

11. Alcon, R. R., and Mulford, R. N., AIP Conf. Proc. 370, pp. 1057-1060, (1996).

12. Mulford, R. N., and Swift, D. C., AIP Conf. Proc. 706, pp 875-878 (2004).

13. Mulford, R. N., Swift, D. C., Collyer, A. M., and White, S. J., Proc. of the HDP V, St Malo, France, pp. 81-90, (2003).

14. Maienschein J. L, and Garcia, F., Thermochim. Acta, 384, pp 71-83 (2002). 